\title{
Sustain Analysis Based On Features Identification In Bioinformatics Tools
}

\author{
Jayanthi Manicassamy and P. Dhavachelvan
}

\begin{abstract}
Today, there is a remarkable incremental development in all areas including bioinformatics for tools and technologies. The reason behind this progress includes various reasons like structural analysis, sequence analysis, functional analysis etc. In this perspective, there is a need for best tools development or utilization with better performance and of easy maintenance. Since the area of bioinformatics involves in solving real world problems there is a vast development of tools and technologies. For better analysis of selective tools with better performance and maintenance it is necessary to make analysis based evaluation that could be carried out automatically for reducing time and resources. Taking these aspects into consideration, a tool have been developed for identifying features from the tools document for analyzing the scope, sustain and evaluation that could be carried out. Since ontology plays a vital role for identifying real world thing it has also been incorporated in this tools which have been narrated in this paper. Based on the features identified scope of the each tools are assessed for possible evaluation that could be carried out on each tool for performance evaluation by graph plotting. Apart from this based on the scope sustain analysis is approximately carried out on the tools automatically. In this paper theoretical description of developed tool evaluation have also been represented which found to be good by means of reducing time, resource and effort.
\end{abstract}

Index Terms - Bioinformatics, Information Extraction, Ontology, Evaluation, Software Metrics.

\section{INTRODUCTION}

Bioinformatics is merging of computational biology with few other sciences which is currently a developing field. The area found to be lacking in development previously but now this area found to have tremendous development comparatively to other area. Solution provided in computational bases for biological problems through functional analysis, sequence analysis, structural analysis etc. This solution provided a way for tremendous development of various tools and technological for solving real world problems [1-4]. Today the desire and need for development especially new tool development cannot be evaded whose analyses are based on tools features and functionalities [5, 6]. Tools analysis is mainly for recognizing the pitfalls that could be overcome for further development or evading from utilization [7]. Selecting tools and evaluating tools and analyzing it's sustain by means of functionality is a tedious task, requiring vast time. In this viewpoint there is a need for

Manuscript received August 5, 2009.

Jayanthi Manicassamy and P. Dhavachelvan are from Department of Computer Science, Pondicherry University, Kalapet, Pondicherry, India. (E-mail: jmanic2@yahoo.com, dhavachelvan@gmail.com). development for making the task easier and time consuming one.

An automatic tool for identifying and extracting features have been developed for assessing possible measures for carrying out performance evaluation and to analyze the sustain of the tools which laid as a solution to the represented problems. Ontology has been incorporated for providing a better solution which has been narrated in this paper. In section II architecture and functioning methodology have been explained. Where as, in section III carried out evaluation have been explained.

\section{ARChitecture And Functioning Methodology}

In this section description on overall architectural and functioning methodology for the developed tool have been represented which involves features identification and scope level analyzing for carrying out possible measures for evaluating tools performance. Sustain analysis which is recognized approximately based on the features identification made.

\section{A. Architecture}

Here the architecture of the developed automated tool has been represented in figure 1. The processes involved for extracting features from the tool document submitted from which keywords are extracted using Ontology Phaser for domain specific. The extracted keywords are stored in temp file replaced with associated words using Association Locator and database. Based on the features identified using database scope of the tool is analyzed based on which the tool is weighted. Apart from this possible evaluation measures for the tool for performance analysis is identified by utilizing the database is done by the Evaluator. Sustainer is responsible for sustain analyses the maintenance requirement and its level from the identified. Organizer process is to organize the extracted information in a structured form by categorizing, scope and its level, possible performance evaluation measures and sustain details. Graph plotter is responsible for possible metrics for evaluating the specific tools performance, scope level of the tool with tools sustain level.

\section{B. Functioning Methodology}

In this section the processing methodology for the developed tool have been explained through procedural approach for identifying features for carrying out analysis and by displaying the assessed result in graph and structure form 


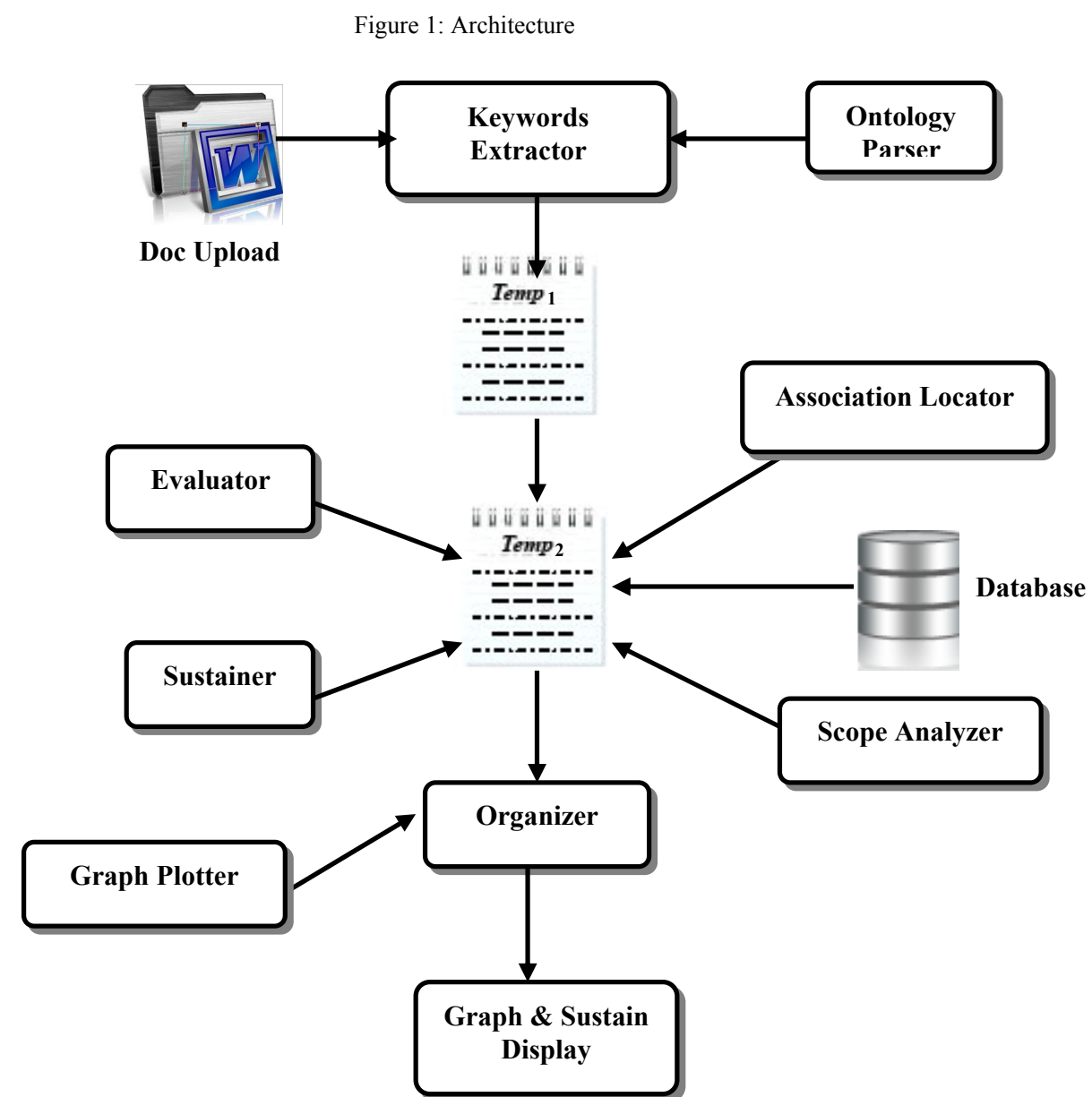

have been narrated. Database of the tool consists of predefined set of features, scope and standard metrics by utilization majority of the task get completed. In the tool development we have incorporated ontology for the specified domain specific for identifying features of the existing tools for the uploaded document which is one of the main activities involved.

\section{A. Procedural Approach}

Word document file is upload which is considered to be the input for this tool which the only position where human intervention is required where as other activities is automatic. The uploaded file is fully scanned through Keywords Extractor using Ontology Phaser for keywords identification based both on domain and non-domain bases which is stored in Temp $_{1}$ file. From extracted keywords using Association Locator features are identified from the database are represented in $\mathrm{Temp}_{2}$ file. Scope Analyzer is responsible for identifying the scope of the tool based on the features identified, apart from this tool scope level is evaluated by

$$
\sum\left(\mathrm{F}_{\mathrm{S}} * 2\right)+\left(\mathrm{P}_{\mathrm{S}} * 1.5\right)
$$

Where, $F_{S}$ is full utilization of scope and $P_{S}$ is partial utilization of scope. If the scope level is less than or equal to 3 , then the scope is considered to be less. . If the level is greater than 3 but less than or equal to 5 , then the scope is considered to be normal and if the level is greater than 5 then the scope is considered to be broader. Evaluator is responsible for identifying possible set of metrics for carrying out the tools performance from the database based on the identified features. Sustainer activity is to identify the level of maintenance easy, better and hard based on the features identified and its requirements through utilization of database. Organizer is responsible for plotting graph using Graph Plotter and displaying sustains details. Figure 2 and figure 3 represents the graph output of the inputted tool document. Table 3 is the representations of the tool sustain.

\section{EXPERIMENTAL RESUlts BASED EVALUATION}

Various experiments have been carried out on this tool by uploading various tools document and extracting features apart form this manually extraction have been done from the study of the document which we have discussed in this section. The results have been analyzed based on experiments carried out and is evaluated in terms of time, resources and effort.

\section{A. Time}

The tool found to be good in consuming time since the tool is an automatic one where the only place where the human intervention is required is uploading the tool document. Since are the required identifications like scope, performance evaluation and tool sustain is automatically done, it is clearly viewable that the time consumption from tool assessment is very less. 


\section{B. Resources}

Apart from time consumption resource consumption is utilizing the developed tool found to be good in effort reduction since manual effort for effort identification, metrics

Figure 2: Scope level Representation

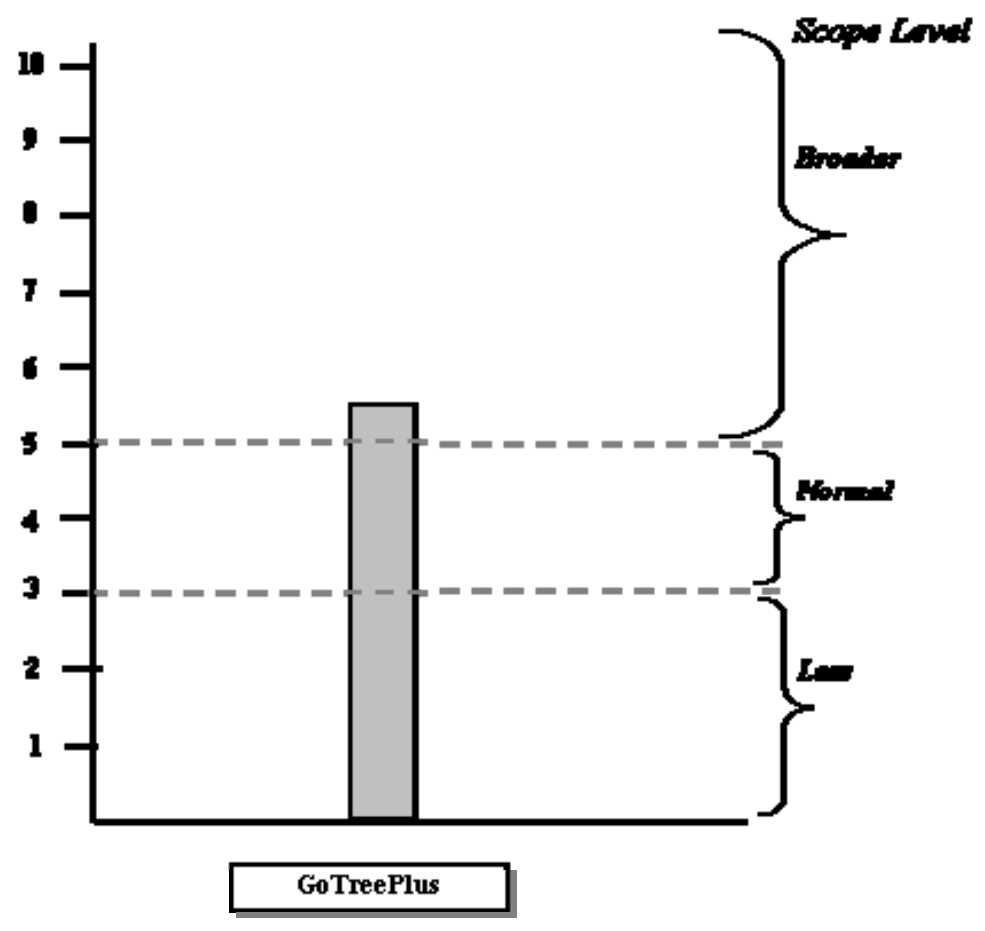

Figure 3: Performance Evaluating Metrics

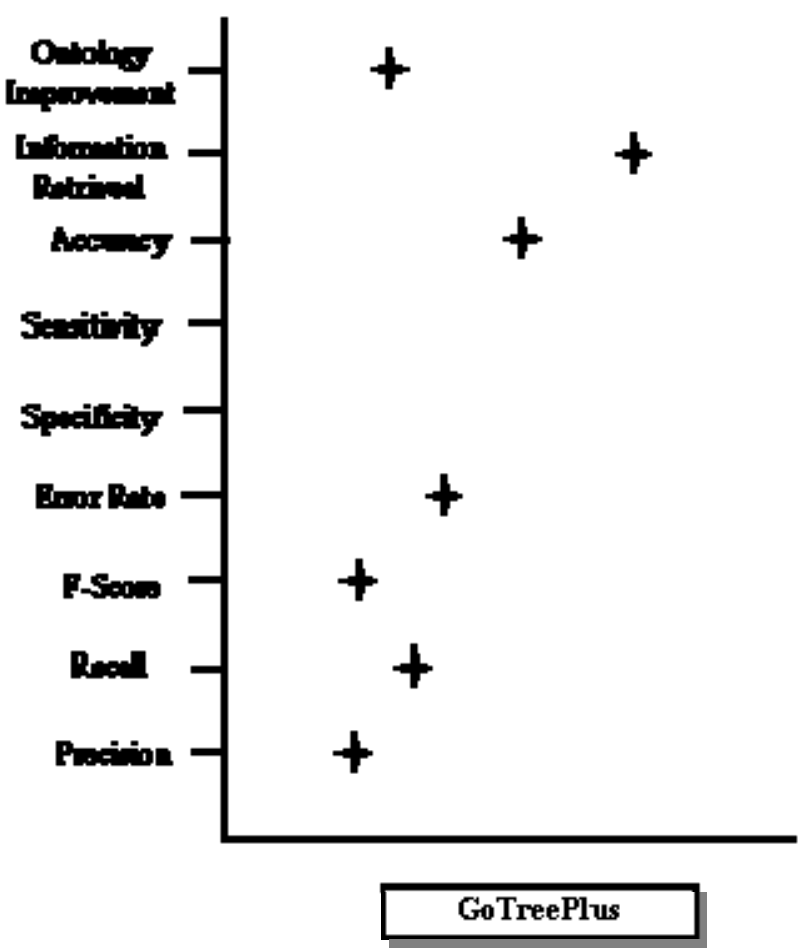

also very less in terms of human resources. Since the human intervention is only involved in uploading the documents. The tool is also found to be cost effective since human resource consumption is less and tool is freeware.

\section{Effort}

Identifying features and functionalities for the tools by identification and analyzing tool sustain. Here effort in terms of time found to have a tremendously reduced by utilizing the tools where there is vast requirement of time for features, performance metrics identification and for arriving at a conclusion for tool sustain. 
TABLE 1: SUSTAIN REPRESENTATION

\begin{tabular}{|l|c|c|}
\hline \multicolumn{1}{|c|}{ Sustain } & $\begin{array}{c}\text { GoTreePlu } \\
\text { s }\end{array}$ & Details \\
\hline Stability & $\sqrt{ }$ & - \\
\hline New Requirements & $\sqrt{ }$ & .Net, Windows Platform \\
\hline Upgradeable & $\sqrt{ }$ & \\
\hline Cost Effective & $\sqrt{ }$ & \\
\hline Internet Based & $\sqrt{ }$ & \\
\hline Mobility & X & \\
\hline Sustain Level & Easier & \\
\hline
\end{tabular}

\section{CONCLUSION}

The main aim of the paper is to provide a description about the developed automated tool for bioinformatics tool features identification based on the document submitted as input. Set of process have been carried out for efficient usage of ontology which has been incorporated in this paper. Extracted information based on keyword, features are identified for identifying the tools scope and evaluating the scope level. Apart from this based on the identification made possible metrics for tools performance evaluation is identified and tool sustain is analyzed in terms of stability, new requirements, upgradeability etc. The reason behind this development is to reduce effort through automatic tools evaluation rather than manual analysis. The limitation doesn't stop to this and the results observed based on the evaluation carried out in terms of effort, time consuming and resource consuming found to be good.

\section{REFERENCES}

[1] Jochen Farwer, Martin J. Packer, Christopher A. Hunter, "PREDICTOR: A Web-Based Tool for the Prediction of Atomic Structure from Sequence for Double Helical DNA with up to 150 Base Pairs", ISO Press, 2007.

[2] Xiang Xu, Jinyu Wu, Jian Xiao, Yi Tan, Qiyu Bao, Fangqing Zhao and Xiaokun $\mathrm{Li}$, "PlasmoGF: an integrated system for comparative genomics and phylogenetic analysis of Plasmodium gene families", ACM Portal, pp 1217-1220, March 2008.

[3] Maryan E.Martone, Joshuna Tran, Willy W. Wong, Joy Sargis, Lisa Fong, Stephen Larson, Stephan P. Lamont, Amarnath Guptha, Mark H. Ellisman, "The Cell Centered Database Project : An updated on building community resources for managing and sharing $3 \mathrm{D}$ imaging data", Elsevier, October 2007.

[4] Francois Belleau, Marc-Alexandre Nolin, Nicole Tourignly, Philippe Rigault, Jean Morissette, "Bio2RDF: Towards a mashup to build bioinformatics knowledge systems", Elsevier, September 2007.

[5] Yang Jin, Ryan T McDonald, Kevin Lerman, Mark A Mandel, Steven Carroll, Mark Y Liberman, Fernando C Pereira, Raymond S Winters, Peter S White, "Automated recognition of malignancy mentions in biomedical literature", November 2006

[6] Rolf BackofenJen, Liviu BadeaBuc, Pedro BarahonaLis, Albert BurgerEdi, McLeodEdi, Steffen M"ollerRos, Werner NuttEdi, Bjorn OlssonSko, Michael SchroederDre, Sylvain SolimanPar, He TanLin, Doina TiliveaBuc, Sebastian WillJen, "Usage of Bioinformatics Tools and Identification of Information Sources", 2005.

[7] Jayanthi Manicassamy and P. Dhavachelvan, "Based Accuracy Perpetuation for Bioinformatics Sequence Analysis Tools", International Journal of Recent Trends in Engineering (IJRTE) pp 550-555, May 2009.

[8] Michael Baitaluk, Mayya Sedova, Animesh Ray and Amarnath Gupta, "BiologicalNetworks: visualization and analysis tool for systems biology", Oxford Journal, pp W466-W471, March 2006.

[9] Matteo Fattore and Patrizio Arrigo, "Knowledge Discovery and System Biology in molecular medicine: an application on neurodegenerative diseases", ISB, December 2004.
[10] Giorgio Valentini and Nicolo Cesa Bianchi, "HCGene: a software tool to support the hierarchical classification of genes", ACM Portal, pp 729-731, January 2008.

[11] Daniele Masotti, Christine Nardini, Simona Rossi, Elena Bonora, Giovanni Romeo, Stefano Volinia and Luca Benini, "TOM: enhancement and extension of a tool suite for in silico approaches to multigenic hereditary disorders", ACM Portal, pp 428-429, November 2007.

[12] Simon Gog, Martin Bader and Enno Ohlebusch, "GENESIS: genome evolution scenarios", ACM Portal, pp 711-712, January 2008.

[13] Stephen A. Smith and Casey W. Dunn, "Phyutility: a phyloinformatics tool for trees, alignments and molecular data", ACM Portal, pp 715-716, January 2008.

[14] Michael Hackenberg and Rune Matthiesen, "Annotation-Modules: a tool for finding significant combinations of multisource annotations for gene lists", ACM Portal, pp 1386-1393, April 2008.

[15] Weil Lai, Vidhu Choudhary and Peter J. Park, "CGHweb: a tool for comparing DNA copy number segmentations from multiple algorithms “, ACM Portal, pp 1014-1015, February 2008.

[16] J.W. Ely, J.A. Osheroff, M. H. Ebell, "A taxonomy of generic clinical questions: classification study”, Brit. Med. J. 321, 2000, pp 429-432. 